\title{
A three-dimensional numerical model for incompressible two-phase flow of a granular bed submitted to a laminar shearing flow
}

\author{
Julien Chauchat and Marc Médale \\ IUSTI CNRS UMR 6595 - Polytech'Marseille - Aix-Marseille Université (U1), \\ 5 rue Enrico Fermi, 13453 Marseille cedex 13, France
}

\begin{abstract}
A numerical model for the simulation of incompressible two-phase flows of a flat granular bed submitted to a laminar shearing flow is presented, considering a two-fluid model and a mixed-fluid one. The governing equations are discretized by a finite element method and a penalisation method is introduced to cope with the incompressibility constraint. A regularisation technique is used to deal with the visco-plastic behaviour of the granular phase. Validations are carried out on three flow test cases: a Bingham fluid between two infinite parallel planes, a Bingham fluid in a square lid-driven cavity and a Newtonian fluid over a granular bed in a two-dimensional configuration, for which we compare our numerical results with existing analytical or numerical results. The accuracy and efficiency of the numerical models have been compared for the two formulations of the two-phase flow model. It turns out that the two-fluid model requires ten times more CPU time than the mixed-fluid one for a comparable accuracy, which can be achieved provided one takes a smaller regularisation parameter in the latter model. Finally, three-dimensional computations are presented for the flow of a Newtonian fluid over a granular bed in a square and circular cross-section ducts.
\end{abstract}

Key words:

Two-phase flow model, Fluid-particle coupling, Viscoplastic flow, Regularisation technique, Quadratic Finite element

\section{Introduction}

Particles transport occurs in a variety of environmental and industrial flows such as sediment transport in rivers or at coasts, hydrate formation in pipelines (oil production) or granular transport in food or pharmaceutical industries. There are mainly two modes for particles to be transported by a flow: suspended-load or bed-load. Suspended load is the part of the load where particles are carried without contact with the bed. On the other way, bed-load is the part of the load that is carried with intermittent contact with the bed, by rolling, sliding and bouncing [15]. In this paper, we focus only on the bed-load transport and more precisely in laminar flow conditions.

The bed-load transport is by nature a two-phase problem (fluid-particles). The particles of the bed are moving depending on the value of the shear stress exerted by the fluid, the shear stress being usually made dimensionless by 
the apparent weight of a single particle the so-called Shields number $\theta$ [31]. If the Shields number based on the fluid bed shear stress is lower than a critical value $\theta_{c}$ the particle flux is zero otherwise it evolves as a function of $\theta$ and $\theta_{c}[13,4,32,11,28]$. The bed-load is usually modelled as a two-layer problem: a pure fluid layer at the top and a fluid-particles mixture layer at the bottom, the two layers being separated by the bed upper surface. The fluid motion in the upper layer is solved assuming weak interactions between the two layers. From this calculation the fluid bed shear stress is known and the particle flux in the lower layer is deduced from an algebraic relationship. A different approach for the bed-load has been proposed by Ouriemi et al. [26]. The authors have proposed some closures of the two-phase model which are appropriate to a situation in which the sediment can be considered as a mobile granular medium where the particles are in contact: the interphase force is then Darcy drag and buoyancy, the fluid phase stress is of Newtonian form, and the particle phase stress is described by a granular rheology (Coulomb friction).

The granular rheology shares some properties with visco-plastic rheology, in particular it exhibits a threshold of motion due to the friction between grains. The archetype model for visco-plastic material is the Bingham model [9]. It is possible to identify the Coulomb friction model for the granular media with the Bingham model in which the fluid viscosity vanishes. The simulation of Bingham fluid flows have been the subject of many papers in the literature (see [12] for a recent review). There are mainly two approaches to deal with the yield stress in the Bingham model: the Augmented Lagrangian technique $[14,17]$ and the regularisation technique $[8,29,16]$. In the Augmented Lagrangian technique, the discontinuity in the Bingham constitutive relationship is treated by introducing a new primal variable and a Lagrange multiplier that enforces it to be equal to the strain rate tensor. This method is particularly accurate to capture and predict the yielded regions of the flow. In the regularisation approach, the Bingham viscosity is "regularised" by adding a small quantity to the magnitude of the rate-of-strain tensor in the denominator. The solid regime is replaced by a very viscous one. But the cost overrun for the Augmented Lagrangian Methods compared with regularization one is obvious in terms of memory due to the introduction of an additional tensor variable (the "true" strain rate), whereas in terms of CPU time the comparison is not known a priori. Actually, the regularized problem is equivalent to the flow of a shear thinning material that induces additional non-linearity in the equations. The computation of which can significantly increase the CPU time and do not allow to conclude on the most efficient method. However, let us mention that the implementation of the regularization method is easier than the Augmented Lagrangian one. Therefore we choose the regularisation technique to deal with the yield stress in our two-phase flow model. This method is advantageous for its simplicity but one must be careful of the induced creeping flow in the yielded regions that arises when using regularisation.

In this paper we present a three-dimensional Finite Element Method (FEM) model of the two-phase incompressible flow model for bed-load transport presented by Ouriemi et al. [26]. Our first concern is to propose a numerical model able to predict accurately the bed-load transport in laminar shearing flows. It is restricted to the cases where the granular bed does not change its shape in the course of time, consequently ripples and dunes formation are beyond the scope of this paper. We have considered two formulations of the two-phase model. In the two-fluid model the unknowns are the velocities and pressure in each phase (fluid and particles) whereas in the mixed fluid model the 
fluid-particles mixture is only considered assuming that fluid and particles have the same velocity. The computational efficiency of the numerical models associated with both formulations is investigated in in terms of accuracy, CPU time and memory usage. The two-phase flow model equations and the numerical modelling are presented in Section 2. Section 3 is devoted to the validation of the model by comparison with analytical solutions or published numerical results. Firstly, we have validated the numerical model for Bingham fluid flows on two test cases. We have compared the numerical model with an analytical solution for the flow of a Bingham fluid between two infinite parallel planes. We have also compared our model with the numerical results of Mitsoulis and Zisis [24] for the flow of a Bingham fluid in a square lid-driven cavity. Then we have validated the numerical model with the analytical solution presented by Ouriemi et al. [26] for the flow of a Newtonian fluid over a granular bed in a two-dimensional configuration. After the validation, we present in Section 4 the application of the two-phase model to simulate the bed-load transport in threedimensional configurations, a square cross-section and a circular cross-section ducts. Finally, we give concluding remarks in section 5 .

\section{The two-phase flow model}

Following Ouriemi et al. [26], we present here the formulation of the two-phase flow model for bed-load transport in laminar shearing flows.

\subsection{Mathematical formulation}

\subsubsection{Governing equations}

Given a cartesian coordinate system $(O, x, y, z)$ where $x$ represents the stream-wise direction, $y$ the lateral direction and $z$ the vertical upward direction, the velocity vector of the $\mathrm{k}$ phase and its cartesian components are respectively denoted by $\overrightarrow{u^{k}}=\left(u^{k}, v^{k}, w^{k}\right)$. $\mathrm{k}$ is taken to be $f$ for the fluid phase and $p$ for the particulate one. We start from Jackson's equations [21] to get the set of governing equations for the two-phase problem.

For the fluid phase, the continuity equation reads:

$$
\frac{\partial \epsilon}{\partial t}+\nabla \cdot\left(\epsilon \overrightarrow{u^{f}}\right)=0
$$

where $\epsilon$ designates the volume fraction of the fluid phase. The particulate phase continuity equation has the same form:

$$
\frac{\partial \phi}{\partial t}+\nabla \cdot\left(\phi \overrightarrow{u^{p}}\right)=0
$$

where $\phi$ is the particulate phase volume fraction. The global volume conservation imposes $\phi+\epsilon=1$.

The momentum equations for the fluid and particulate phases are respectively:

$$
\rho_{f}\left[\frac{\partial \epsilon \overrightarrow{u^{f}}}{\partial t}+\nabla \cdot\left(\epsilon \overrightarrow{u^{f}} \otimes \overrightarrow{u^{f}}\right)\right]=\nabla \cdot\left(\overrightarrow{\overline{\sigma^{f}}}\right)-n \vec{f}+\epsilon \rho_{f} \vec{g},
$$




$$
\rho_{p}\left[\frac{\partial \phi \overrightarrow{u^{p}}}{\partial t}+\nabla \cdot\left(\phi \overrightarrow{u^{p}} \otimes \overrightarrow{u^{p}}\right)\right]=\nabla \cdot\left(\overline{\overline{\sigma^{p}}}\right)+n \vec{f}+\phi \rho_{p} \vec{g}
$$

where $\overline{\overline{\sigma^{f}}}$ and $\overline{\overline{\sigma^{p}}}$ represent the stress tensor associated with the fluid and particulate phases respectively. $\overrightarrow{n f}$ represents the average force exerted by the fluid on the particles and $\vec{g}$ is the gravity acceleration vector.

The set of partial differential equations (1)-(4) introduces more unknowns than the number of equations then closure relationships are needed to solve the problem. These relations are of two types: fluid-particle interactions and stress tensor expressions.

\subsubsection{Closures}

Interaction term. Following Jackson [21] the average force exerted by the fluid on the particles can be decomposed in two contributions. The first one corresponds to the generalized buoyancy force and the second one gathers all the remaining contributions.

$$
\overrightarrow{n f}=\phi \nabla \cdot\left(\overline{\overline{\sigma^{f}}}\right)+n \overrightarrow{f^{1}}
$$

For a viscous fluid flow in a porous media, the remaining contributions reduce to the viscous drag force due to the relative motion between phases. Using the Darcy law, the term $n \vec{f}$ can be written:

$$
n \overrightarrow{f^{1}}=\eta \frac{\epsilon^{2}}{K}\left(\overrightarrow{u^{f}}-\overrightarrow{u^{p}}\right)
$$

where $\eta$ is the dynamic viscosity of the pure fluid. The coefficient of permeability is empirically linked to $\epsilon$ and the particle diameter $d$ by the Carman-Kozeny relationship:

$$
K=\frac{\epsilon^{3} d^{2}}{k_{C K}(1-\epsilon)^{2}}
$$

A typical value for $k_{C K} \approx 180$ is proposed by Happel and Brenner [20] and Goharzadeh et al. [18].

Stress tensors. The fluid phase has been assumed to be a Newtonian viscous liquid in which the Einstein dilute viscosity formula has been chosen to be applied to the concentrated situation :

$$
\overline{\overline{\sigma^{f}}}=-p^{f f} \overline{\bar{I}}+\overline{\overline{\tau^{f}}}=-p^{f} \overline{\bar{I}}+\eta_{e}\left(\nabla \overrightarrow{u^{m}}+\left(\nabla \overrightarrow{u^{m}}\right)^{T}\right) \text {, }
$$

where $\eta_{e}=\eta(1+5 \phi / 2)$ is the effective viscosity of the mixture and $\overrightarrow{u^{m}}=\epsilon \overrightarrow{u^{f}}+\phi \overrightarrow{u^{p}}$ is the velocity of the mixture.

The particle phase has been assumed to be described by Coulomb solid friction in which the extra stress is proportional to the particle pressure.

$$
\overline{\overline{\sigma^{p}}}=-p^{p} \overline{\bar{I}}+\overline{\overline{\tau^{p}}}
$$

In the frame of the three-dimensional model we express the Coulomb friction model in tensorial form following the idea of Jop et al. [22]:

$$
\overline{\overline{\tau^{p}}}=\eta_{p}\left(\left\|\overline{\dot{\bar{\gamma}}^{p}}\right\|, p^{p}\right) \overline{\overline{\dot{\gamma}^{p}}}
$$


with

$$
\eta_{p}\left(\left\|\overline{\overline{\dot{\gamma}}^{p}}\right\|, p^{p}\right)=\frac{\mu p^{p}}{\left\|\overline{\overline{\dot{\gamma}^{p}}}\right\|}
$$

where the rate of strain tensor $\overline{\overline{\dot{\gamma}^{p}}}$ is defined as $\overline{\bar{\gamma}^{p}}=\nabla \overrightarrow{u^{p}}+\left(\nabla \overrightarrow{u^{p}}\right)^{T}$ and its magnitude is given by the square root of its second invariant $\left\|\overline{\dot{\bar{\gamma}}^{p}}\right\|=\sqrt{\frac{1}{2} \operatorname{Tr}\left(\overline{\dot{\gamma}}^{2}\right)}$. We emphasise that this expression in the one dimensional case reduces to the classical Coulomb expression: $\tau_{x z}^{p}=\mu p^{p}$.

\subsubsection{Dimensionless equations}

In the preceding subsections we have presented the ingredients of the two-phase model for bed-load transport, we summarise here the model equations to be solved. We consider two formulations of the model. The first one, called the two-fluid model, is based on the solution of mass and momentum conservation equations for each phase (12). The second one, called the mixed-fluid model, is based on the solution of mass and momentum equations for the mixture (13) (i.e.: a single effective phase is considered). In this latter formulation, the mass and momentum equations are simply obtained by summing the corresponding equations over each phase (fluid and particles).

In the following, we assume that the volume fractions are constant in space and time meaning that the interface between the fluid-particle mixture and the pure fluid region is fixed and no dilatation occurs. This assumption implies that fluid and particulate phase as well as the mixture are incompressible. We also express the equations for the fluid phase in terms of the mixture velocity.

Following Ouriemi et al. [26] we make all the values dimensionless by scaling the length by $\mathrm{H}$, the height of the flow, and the stresses by $\Delta \rho g H$, and therefore the time by $\eta / \Delta \rho g H$ where $\Delta \rho=\rho_{p}-\rho_{f}$. Using these scales one obtains the following dimensionless equations for the two formulations of the two-phase model.

\section{Two-fluid model.}

$$
\left\{\begin{aligned}
\nabla \cdot\left(\overrightarrow{u^{m}}\right) & =0 \\
\nabla \cdot\left(\overrightarrow{u^{p}}\right) & =0 \\
G a \frac{H^{3}}{d^{3}} \frac{D \overrightarrow{u^{m}}}{D t} & =-\nabla p^{f}+\nabla \cdot\left(\frac{\eta_{e}}{\eta}\left(\nabla \overrightarrow{u^{m}}+\nabla{\overrightarrow{u^{m}}}^{T}\right)\right)-\frac{H^{2}}{K}\left(\overrightarrow{u^{m}}-\overrightarrow{u^{p}}\right)+\frac{\rho_{f} \vec{g}}{\Delta \rho\|\vec{g}\|} \\
G a \frac{H^{3}}{d^{3}} R_{\rho} \phi \frac{D \overrightarrow{u^{p}}}{D t} & =-\nabla p^{p}+\nabla \cdot\left(\frac{\eta_{p}}{\eta}\left(\nabla \overrightarrow{u^{p}}+\nabla{\overrightarrow{u^{p}}}^{T}\right)\right)-\phi \nabla p^{f}+\phi \nabla \cdot\left(\frac{\eta_{e}}{\eta}\left(\nabla \overrightarrow{u^{m}}+\nabla{\overrightarrow{u^{m}}}^{T}\right)\right)+\frac{(1-\phi) H^{2}}{K}\left(\overrightarrow{u^{m}}-\overrightarrow{u^{p}}\right)+\frac{\phi \vec{g}}{\|\vec{g}\|}
\end{aligned}\right.
$$

In these equations, $R_{\rho}=\rho_{f} / \rho_{p}$ represents the density ratio and $G a=d^{3} \rho_{f} \Delta \rho g / \eta^{2}$ is the Galileo number where $d$ is the particle diameter. The Galileo number is a Reynolds number based on the settling velocity of particles. 
Mixed-fluid model.

$$
\left\{\begin{aligned}
\nabla \cdot\left(\overrightarrow{u^{m}}\right) & =0 \\
G a \frac{H^{3}}{d^{3}}\left(1+R_{\rho}\right) \frac{D \overrightarrow{u^{m}}}{D t} & =-\nabla p^{f}-\nabla p^{p}+\frac{\rho_{m} \vec{g}}{\Delta \rho\|\vec{g}\|}+\nabla \cdot\left(\frac{\eta_{e}}{\eta}\left(\nabla \overrightarrow{u^{m}}+\nabla \vec{u}^{T}\right)\right)+\nabla \cdot\left(\frac{\eta_{p}}{\eta}\left(\nabla \overrightarrow{u^{m}}+\nabla{\overrightarrow{u^{m}}}^{T}\right)\right)
\end{aligned}\right.
$$

\subsection{Numerical model}

The Finite Element Method (FEM) leads to the discretisation of the variational formulation of equations (12) for the two-fluid model and on equation (13) for the mixed-fluid model.

\subsubsection{Weak formulations}

Weak formulation for the two-fluid model. Let us define the physical domains associated with the pure fluid and the fluid-particles mixture by $\Omega_{f}$ and $\Omega_{p}$ respectively and their respective boundaries by $\Gamma_{f}$ and $\Gamma_{p}$ (See Figure 1). From the previous system of partial differential equations (12) one obtains the following weak formulation of the momentum equations for the two-fluid formulation [10], [30]:

Find $\overrightarrow{u_{h}^{m}} \in \mathcal{U}_{h}$ and $p^{f} \in Q_{h}$ satisfying (14), (15) and find $\overrightarrow{u_{h}^{p}} \in \mathcal{U}_{h}$ and $p^{p} \in Q_{h}$ satisfying (16), (17) $\forall \overrightarrow{\delta u} \in \mathcal{V}_{h}$ and $\forall \delta p \in Q_{h}$ where

$$
\begin{aligned}
& \mathcal{U}_{h}=\left\{\vec{u} \in H^{1}(\Omega) \mid \vec{u}=\vec{u}_{\text {Dirichlet }} \text { on } \partial \Omega_{\text {Dirichlet }}\right\}, \\
& Q_{h}=\left\{\delta p \in L^{2}(\Omega)\right\} \text {, } \\
& \mathcal{V}_{h}=\left\{\overrightarrow{\delta u} \in H_{0}^{1}(\Omega) \mid \overrightarrow{\delta u}=\overrightarrow{0} \text { on } \partial \Omega_{\text {Dirichlet }}\right\} \text {. } \\
& 0=\int_{\Omega_{f} \cup \Omega_{p}} \delta p \cdot \nabla \overrightarrow{u_{h}^{m}} d \Omega \\
& 0=-\int_{\Omega_{f} \cup \Omega_{p}} G a \frac{H^{3}}{d^{3}} \overrightarrow{\delta u} \cdot \frac{D \overrightarrow{u_{h}^{m}}}{D t} d \Omega-\int_{\Omega_{f} \cup \Omega_{p}} \overrightarrow{\delta u} \cdot \nabla p_{h}^{f} d \Omega+\int_{\Omega_{p}} \frac{H^{2}}{K} \overrightarrow{\delta u} \cdot\left(\overrightarrow{u_{h}^{p}}-\overrightarrow{u_{h}^{m}}\right) d \Omega \\
& -\int_{\Omega_{f} \cup \Omega_{p}} \frac{\eta_{e}}{\eta}\left(\nabla \overrightarrow{\delta u}+\nabla \overrightarrow{\delta u}^{T}\right) \cdot\left(\nabla \overrightarrow{u_{h}^{m}}+\nabla \vec{u}_{h}^{T}\right) d \Omega+\int_{\Omega_{f} \cup \Omega_{p}} \frac{\rho_{f}}{\Delta \rho} \overrightarrow{\delta u} \cdot \frac{\vec{g}}{\|\vec{g}\|} d \Omega \\
& 0=\int_{\Omega_{p}} \delta p \cdot \nabla \overrightarrow{u_{h}^{p}} d \Omega
\end{aligned}
$$




$$
\begin{aligned}
0= & \left.-\int_{\Omega_{p}} G a \frac{H^{3}}{d^{3}} R_{\rho} \phi \overrightarrow{\delta u} \cdot \frac{D \overrightarrow{u_{h}^{p}}}{D t} d \Omega-\int_{\Omega_{p}} \overrightarrow{\delta u} \cdot\left(\nabla p_{h}^{p}-\phi \nabla p_{h}^{f}\right) d \Omega-\int_{\Omega_{p}} \frac{(1-\phi) H^{2}}{K} \overrightarrow{\delta u} \cdot \overrightarrow{u_{h}^{p}}-\overrightarrow{u_{h}^{m}}\right) d \Omega \\
& -\int_{\Omega_{p}} \eta_{p}(\nabla \overrightarrow{\delta u}+\nabla \overrightarrow{\delta u})\left(\nabla \overrightarrow{u_{h}^{p}}+\nabla{\overrightarrow{u_{h}^{p}}}^{T}\right) d \Omega+\int_{\Gamma_{p}} \eta_{p} \overrightarrow{\delta u} \cdot\left(\nabla{\overrightarrow{u_{h}^{p}}}^{T} \nabla{\overrightarrow{u_{h}^{p}}}^{T}\right) \cdot \vec{n} d \Gamma \\
& -\int_{\Omega_{p}} \phi \frac{\eta_{e}}{\eta}(\nabla \overrightarrow{\delta u}+\nabla \overrightarrow{\delta u})\left(\nabla \overrightarrow{u_{h}^{m}}+\nabla{\overrightarrow{u_{h}^{m}}}^{T}\right) d \Omega+\int_{\Gamma_{p}} \phi \frac{\eta_{e}}{\eta} \overrightarrow{\delta u} \cdot\left(\nabla{\overrightarrow{u_{h}^{m}}}^{T}+\nabla{\overrightarrow{u_{h}^{m}}}^{T}\right) \cdot \vec{n} d \Gamma \\
& +\int_{\Omega_{p}} \phi \overrightarrow{\delta u} \cdot \frac{\vec{g}}{\|\vec{g}\|} d \Omega
\end{aligned}
$$

Weak formulation for the mixed-fluid model. For the mixed fluid model, the weak formulation is given by:

Find $\overrightarrow{u_{h}^{m}} \in \mathcal{U}_{h}$ and $p^{f}$ and $p^{p} \in Q_{h}$ satisfying (18) and (19) $\forall \overrightarrow{\delta u} \in \mathcal{V}_{h}$ and $\forall \delta p \in Q_{h}$ where

$$
\begin{aligned}
\mathcal{U}_{h} & =\left\{\vec{u} \in H^{1}(\Omega) \mid \vec{u}=\vec{u}_{\text {Dirichlet }} \text { on } \partial \Omega_{\text {Dirichlet }}\right\} \\
Q_{h}= & \left\{\delta p \in L^{2}(\Omega)\right\} \\
\mathcal{V}_{h}= & \left\{\overrightarrow{\delta u} \in H_{0}^{1}(\Omega) \mid \overrightarrow{\delta u}=\overrightarrow{0} \text { on } \partial \Omega_{\text {Dirichlet }}\right\} \\
0= & \int_{\Omega_{f} \cup \Omega_{p}} \delta p \cdot \nabla u_{h}^{m} d \Omega \\
0= & \int_{\Omega_{f} \cup \Omega_{p}} G a \frac{H^{3}}{d^{3}}\left(1+R_{\rho}\right) \overrightarrow{\delta u} \cdot \frac{D u_{h}^{m}}{D t} d \Omega-\int_{\Omega_{f} \cup \Omega_{p}} \overrightarrow{\delta u} \cdot\left(\nabla p_{h}^{p}+\nabla p_{h}^{f}\right) d \Omega \\
& \quad-\int_{\Omega_{f} \cup \Omega_{p}} \frac{\eta_{p}+\eta_{e}}{\eta}\left(\nabla \overrightarrow{\delta u}+\nabla \overrightarrow{\delta u}^{T}\right)\left(\nabla \overrightarrow{u_{h}^{m}}+\nabla{\overrightarrow{u_{h}^{m}}}^{T}\right) d \Omega+\int_{\Omega_{f} \cup \Omega_{p}} \frac{\rho_{m}}{\Delta \rho} \overrightarrow{\delta u} \cdot \frac{\vec{g}}{\|\vec{g}\|} d \Omega
\end{aligned}
$$

\subsubsection{Algorithms associated with the two formulations}

The first algorithm dealing with the two-fluid formulation is based on the solution of the system (14)-(17) in a weakly coupled way. For each Newton-Raphson iteration we solve for the fluid momentum equation (15) whereas the particulate phase velocity is taken at its previous iteration value. From the value of the mixture velocity obtained at this stage the particulate phase velocity is computed by solving equation (17). On the other hand the algorithm devoted to the mixed-fluid formulation is based on the solution of the system (18-19).

The non-linearities in the governing equations are solved by a Newton-Raphson algorithm by taking the first variation of the variational formulations (15)-(17) and (19). Only the advective terms and the particle stress term (visco-plastic) induce a contribution in the first variation of the variational formulations.

\subsection{Regularisation technique}

The stress tensor for the particulate phase (10) reveals the presence of a yield stress that depends on the granular pressure. The mixed-fluid model (13) can be identified as a Bingham fluid in which the yield stress vary linearly with 
the granular pressure. This remark allows us to use some well-known methods derived for Bingham flows to deal with the visco-plastic behaviour of the fluid-particle mixture.

As with a Bingham model, the particulate viscosity diverges when the shear rate tends toward zero, rising evident numerical problems. The idea of a regularisation technique from a mathematical point of view is to smooth the divergence of the viscosity function. The consequence on the computational behaviour of the yield stress fluid results in a very viscous fluid at zero rate of strain instead of a pure rigid body behaviour. One of the easiest solutions to regularise the viscosity is the following [16]:

$$
\eta(\overline{\dot{\gamma}})=\mu+\frac{\tau_{0}}{\|\overline{\dot{\gamma}}\|+\lambda}
$$

where $\lambda$ is the regularisation parameter $(\lambda<<1)$ and $\tau_{0}$ is the yield stress. We have chosen this expression in our implementation of the numerical model. A review of regularisation technique can be found in Frigaard and Nouar [16].

\subsection{Implementation}

In our implementation, we use piecewise quadratic polynomial approximation for the velocity and piecewise linear discontinuous approximation for the pressure. In the computations, we have employed a 27-nodes hexahedra element (H27) for the velocities. The incompressibility constraint is solved by a penalisation method. The code is developed with the PETSc library $[6,5,7]$ which provides several parallel iterative and direct solvers.

As we use a penalisation method to cope with the incompressibility constraint, all the algebraic systems have been solved by the MUMPS direct solver $[2,1,3]$ with a penalty parameter set to $10^{9}$ for all the simulations presented in this paper.

\section{Validations}

In this section we address a twofold goal for the validation of our numerical model. The first one concerns the implementation of viscoplastic flow using a regularisation technique (subsection 3.1) and the second one deals with the implementation of the two formulations associated with the two-fluid model and the mixed-fluid one (subsection 3.2). In each case we discuss the accuracy and numerical efficiency of the implemented numerical model.

\subsection{Test cases for Bingham fluid flows}

We have begun with test cases on Bingham fluid flows because of the close link that exists between the Coulomb friction for the particulate phase (10) and the Bingham fluid model. These two models exhibit the presence of a yield stress. This is particularly important to validate the implementation of the regularisation on "well-known" configurations (Bingham fluid flows) in order to avoid questions on it when performing two-phase flows simulations. We have done two test cases. The first one is the flow of a Bingham fluid between two infinite parallel planes. The second one is the flow of a Bingham fluid in a square lid-driven cavity. 


\subsubsection{Flow of a Bingham fluid between two infinite plane}

In this first test case we study the flow of a Bingham fluid between two infinite parallel planes. This is a 2D problem where the longitudinal velocity component only depends on the vertical coordinate. This simple problem is particularly interesting for the validation since it possesses an analytical solution for the longitudinal velocity (See Guyon et al. [19] p. 219-220). Figure 2 shows the sketch of the problem and boundary conditions. The simulation are performed using a regularisation method with a regularisation parameter $\lambda=10^{-4}$ and the Bingham number $(B n)$ is equal to 10 .

Figure 3(a) shows the longitudinal velocity profiles for the numerical solution and the analytical one. In order to study the spatial convergence of the numerical model we have considered five meshes. The ratio of the mesh size in the three directions have been kept constant $\Delta x / \Delta z=\Delta x / \Delta y=10$ to avoid errors induced by the distortion of the mesh. The five mesh sizes are reported in Table 1 as well as the CPU time requirements. All the simulations have been performed on a cluster composed of AMD 280 processors at $2.4 \mathrm{GHz}$ and 8 Go of RAM by node. The CPU time evolution with the mesh size is more or less proportional to the number of degrees of freedom. Figure 3(b) shows the Root Mean Square (RMS) error between the numerical solution and the analytical one versus the mesh size (h, defined as an element height $\Delta z$ ).

The longitudinal velocity profiles obtained by numerical simulation are in close agreement with the analytical solution for the five meshes. Figure 3(b) reveals that the spatial order of convergence is approximately of order three which corresponds to the optimal convergence rate of the method (second order approximation) [25, 10, 30, 33]. This particularly good spatial convergence rate can be explained by the fact that the analytic solution is quadratic in the plastic region and constant in the plug located in the central region of the flow. Therefore our tri-quadratic finite element approximation is able to represent at convergence this analytical solution. So, this test case reveals that a correct implementation of the model to simulate Bingham fluid flows as the optimal asymptotic order of convergence has been reached, see figure 3(b).

\subsubsection{Bingham flow in a lid-driven cavity}

The second test case concerns the flow of a Bingham fluid in a square lid-driven cavity. Its main goal is to qualitatively validate the $3 \mathrm{D}$ implementation of the regularisation technique. The sketch of the lid-driven cavity is presented in Figure 4. A tangential velocity is imposed at the top of the cavity whereas the three other boundaries are considered as walls. The geometry is a unit square cavity and the velocity at the top is also chosen to be unity. This test case has been extensively studied and a great amount of literature on viscoplastic flows exists concerning this problem [24, 23, 12]. We have chosen the Mitsoulis and Zisis [24] results as reference who also used a FEM model and a regularisation technique to deal with the yield stress. In the following simulations we have neglected inertial effects (i.e.: $\operatorname{Re}=0)$. The dimensionless number controlling the flow is the Bingham number $\left(B n=\tau_{0} H / \eta U\right)$ where $\tau_{0}$ is the yield stress.

The mesh is composed with 60x1x60 quadratic elements (H27). The regularisation parameter is fixed to $\lambda=$ 
$10^{-4} s^{-1}$. This value is equivalent to the one chosen by Mitsoulis and Zisis [24] but the regularisation technique is different. Mitsoulis and Zisis [24] used the Papanastasiou [29] regularisation whereas we have used the simple regularisation (Cf. 2.3). In the simulations the Bingham number has been considered in the range [0 - 20] to validate our model.

Figure 5 shows the velocity fields in the cavity for various Bingham number $(\mathrm{Bn}=0,2$ and 20$)$. We observe that a rigid region appears at the bottom of the cavity as the Bingham number is increased. Also the velocity decreases as well as the vortex intensity.

Figure 6 shows the yield zone position in the lid-driven cavity for various Bingham number. We compare our results with the results from Mitsoulis and Zisis [24]. The position of the yield zone is defined as the position where the material flows (yields) i.e. : where the magnitude of the stress tensor $\|\overline{\bar{\tau}}\|$ exceeds the yield stress $\tau_{0}\left(\|\overline{\bar{\tau}}\| \geq \tau_{0}\right)$. The results obtained are in good agreement with Mitsoulis and Zisis [24] and validate the implementation of the Bingham model in our FEM model.

As a conclusion on the two Bingham fluid flow test cases one can notice that the regularisation technique implemented give satisfactorily accurate results for Bingham number in the range 0-20 for a regularisation parameter $\lambda=10^{-4}$. Moreover with this numerical parameter a third order (optimal) asymptotic convergence rate has been reached for our tri-quadratic finite element approximation.

\subsection{Two-phase simulation of bed-load transport in $2 D$}

In this subsection we present results on the flow of a Newtonian fluid over a granular bed. The aim of this subsection is first to validate quantitatively the two formulations of the two-phase flow model by comparison with the analytical solution of Ouriemi et al. [26] for the bed-load transport in laminar shearing flows and secondly to assess computational efficiency of the numerical model associated with both formulations.

The sketch of the problem and boundary conditions are given in figure 7. The lower half of the domain is filled with particles at $\phi=0.55$ immersed in a fluid and the upper part is filled with pure fluid $(\phi=0)$. Therefore in this problem the values of the dimensionless numbers are: $R e=210^{-2}, G a=11, R_{\rho}=0.4$ and $d / H=30$. There are several choice for the definition of the Bingham number in the bed-load problem. Actually, in dense granular media the yield stress varies with the normal stress. Therefore one has to choose a pertinent value of the yield stress. Here we choose the yield stress corresponding to the first granular layer, this choice is natural since the relevant length scale for the estimation of the yield stress is the height of the moving granular bed that is of the order of few grain diameters. Assuming a hydrostatic pressure for the granular phase, it reads:

$$
\tau_{0}=\mu_{s} \Delta \rho g d,
$$

where $d$ is the particle diameter. With this yield stress definition the value of the Bingham number in the bed-load problem considered here is $B n=210^{4}$. This value is greatly higher than the one used in the validation tests for Bingham fluid flows ( $\mathrm{Bn} \# 10$ ) and implies accordingly a smaller value of $\lambda$ than the one used in previous Bingham 
flow test cases. So the regularisation parameter is set to $\lambda=10^{-6} s^{-1}$ when its value is not mentioned in the figure captions.

We solved by FEM the two formulations of the two-phase flow model (See subsection 2.1.3) corresponding to the coupled system (15)-(17) for the two-fluid model and equation (19) for the mixed-fluid model. Numerical solutions for the two-fluid model and the mixed-fluid model are presented in figure 8 for a $6 \times 1 \times 40$ mesh with a requested absolute residual lower than $10^{-11}$ per degree of freedom.

Figures $8 \mathrm{a}$ and $8 \mathrm{~b}$ present the comparison of the horizontal velocity profiles obtained for the two formulations of the two-phase flow model (two-fluid and mixed-fluid) by numerical simulations compared with the analytical solution proposed by Ouriemi et al.[26]. In both figures the black solid line represents the analytical solution. A good agreement can be noticed between the numerical results and the analytical solution which validate the FEM model for the bed-load transport in a $2 \mathrm{D}$ configuration.

Moreover we have performed a spatial convergence analysis for both formulations (see figure 9). Concerning the two-fluid model solution the spatial convergence is of order one. Two hypotheses could explain the first order convergence, the presence of a predominant Darcy term in the momentum equations and the regularisation technique applied to the particulate phase rheology. Moreover one can observe that the error on the particulate phase velocity is greater than the fluid one. Therefore the difference could be attributed to the regularisation technique that can be reduced using a smaller regularisation parameter $\lambda$. For the mixed-fluid model solution a third order spatial convergence is reached for the coarsest meshes where the discretisation error dominates. On the other hand for finer meshes the order of convergence reduces and tends to a first order one in the region where it is dominated by the modelling error introduced by the regularisation technique. One can also notice that the RMS error is one order of magnitude greater than for the two-fluid model solution for the same regularisation parameter as in the two-fluid model. Reducing the regularisation parameter $\lambda$ by one order of magnitude for the mixed-fluid model reduces the error accordingly (see figure 9). Therefore the same accuracy as the two-fluid model is recovered but with a tenfold CPU time reduction for the mixed-fluid model compared with the former formulation (see Tab. 2). We point out that the CPU time for the mixed-fluid model with a regularisation parameter $\lambda=10^{-7}$ is only twenty percent higher than the one with $\lambda=10^{-6}$ whereas the error is reduced by one order of magnitude. More surprisingly, it takes the same CPU time for two uniformly refined meshes for a comparable accuracy solution (see Tab. 2). Indeed the iteration count for the finest mesh is five times less than for the coarser meanwhile the CPU time per iteration is five times higher.

Consequently the two-fluid model is much more expensive (ten times in CPU time) than the mixed-fluid one for a comparable accuracy provided one takes a regularisation parameter sufficiently small (one order of magnitude smaller than in the two-fluid model). But one should keep in mind that the mixed-fluid model is based on the strong assumption of zero relative velocity between the fluid and particulate phases, which could be restrictive in actual problems.

It turns out from the results obtained in this section that the modelling error associated with the implemented regularisation technique is connected to the regularisation parameter value $\lambda$. Therefore in order to achieve compu- 
tations with controlled accuracy we suggest to link the value of the regularisation parameter to the Bingham number according to the following empirical relationship: $\lambda=\min \left(10^{-4}, \frac{10^{-4}}{\mathrm{Bn}}\right)$.

\section{Two-phase simulation of bed-load transport in 3D configuration}

We have shown in the last section the convergence of the two-phase numerical model compared with analytical solution for a two-dimensional configuration. We now apply the model to three-dimensional configurations: a square and a circular cross-section ducts. As in the previous test case the values of the dimensionless numbers are: $R e=$ $210^{-2}, G a=11, R_{\rho}=0.4, d / H=30$ and $B n=210^{4}$. However the characteristic length in the definition of the dimensionless numbers is the side length for the square duct whereas it is the diameter in the cylindrical one.

\subsection{Square section duct}

We have performed simulations for a square cross-section duct with two meshes: 6x20x40 and 6x40x80 elements uniformly distributed, only the half of the domain has been solved in the transverse direction for obvious symmetry reasons. Figure 10 shows the velocity profile in a cross section of the duct. The contour colors represent the $\mathrm{x}$-velocity of the mixture $\left(u^{m}\right)$. The horizontal thick solid line at $z=0.5$ represents the position of the granular bed. The fluid and the mixture are sheared in both $\mathrm{z}$ and $\mathrm{y}$ directions inducing an increase in the friction compared with the twodimensional case. Due to this shear increase the velocity is lower than in the two-dimensional case. In order to test the convergence of the solution with respect to the mesh we present in figure 11 the comparison of the velocity profiles on the plane of symmetry of the duct $(y=0)$ for the two meshes. The numerical solutions superimpose themselves except in the neighbourhood of the yield zone where the finest mesh better resolves the yield zone. Figure 12 shows the velocity profiles of the fluid phase velocity in blue and the particulate phase velocity in red (an offset of $10^{-3}$ has been added to make the particulate phase velocity visible) obtained with the two-fluid model for the square cross-section duct $(6 \times 20 \times 40)$. This illustrates the good behaviour of the numerical model in this three-dimensional configuration.

\subsection{Circular section duct}

As for the square cross-section duct we have performed two simulations on the circular cross-section duct. The mesh sizes are 6x896 and 6x3596 quadratic elements in the x direction and in the cross-section respectively. Figure 13 shows the velocity profile in a cross-section of the duct (6x896). As for the square cross-section duct, the hick solid line at $z=0$ represents the position of the granular bed and the contour colors represent the mixture velocity $\left(u^{m}\right)$. Here again the friction is increased in this geometry compared with the two-dimensional configuration. We have compared the velocity profiles on the vertical plane of symmetry to show the convergence of the solution with respect to the mesh size (See figure 14). Again figure 15 illustrates the good behaviour of the numerical model for three-dimensional flow configurations. 


\section{Concluding remarks}

In conclusion, we have developed a numerical model to simulate incompressible two-phase flow of a Newtonian fluid over a granular bed. The model is based on a penalisation method for incompressible flows and a regularisation technique for the visco-plastic behaviour of the granular phase. Validations have been carried out on three test cases: the flow of a Bingham fluid between two infinite parallel planes, the flow of a Bingham fluid in a square lid-driven cavity and the flow of a Newtonian fluid over a granular bed. One can notice the very good agreement between the computations and the existing analytical solutions [19, 26] or numerical results [24]. To get these results we have considered that the modelling error associated with the implemented regularisation technique should be correlated to the regularisation parameter value $\lambda$ according to the empirical relationship: $\lambda=\min \left(10^{-4}, 10^{-4} / B n\right)$.

Concerning the two-phase flow formulation of the bed-load problem we have shown that the mixed-fluid model is computationally more efficient than the two-fluid one (roughly ten times faster and requires $20 \%$ less memory). Moreover, in order to achieve a comparable accuracy of the two models one has to choose a regularisation parameter ten times smaller for the mixed-fluid model than for the two-fluid one. But one should recall that the mixed-fluid formulation is based on the strong assumption that the fluid-particles relative velocity is negligible which could limits its validity range to the cases of small particulate Reynolds number.

Finally we have performed three-dimensional numerical simulation of the bed-load transport in a square and a circular cross-section ducts illustrating the capability of the model to deal with arbitrary geometries where no analytical solution exists. Future developments will concern the implementation of a numerical technique to simulate the motion of the fluid-granular bed interface. Our final goal is to perform three-dimensional simulations for the formation of ripples and dunes that are observed in experiments [? ].

\section{Acknowledgements}

We would like to thank P. Aussillous and É. Guazzelli for fruitful discussions regarding the two-phase flow model and Y. Forterre and O. Pouliquen for discussions regarding the granular rheology. The authors also acknowledge Y. Jobic for technical support. Funding from Agence Nationale de la Recherche (Project Dunes ANR-07-3_18-3892) is gratefully acknowledged.

\section{References}

[1] Amestoy, P. R., Duff, I. S., Koster, J., L’Excellent, J.-Y., 2001. A fully asynchronous multifrontal solver using distributed dynamic scheduling. SIAM Journal on Matrix Analysis and Applications 23 (1), 15-41.

[2] Amestoy, P. R., Duff, I. S., L’Excellent, J.-Y., 2000. Multifrontal parallel distributed symmetricand unsymmetric solvers. Comput. Methods Appl. Mech. Eng. 184, 501-520.

[3] Amestoy, P. R., Guermouche, A., L'Excellent, J.-Y., Pralet, S., 2006. Hybrid scheduling for the parallel solution of linear systems. Parallel Computing 32 (2), 136-156.

[4] Bagnold, R. A., 1956. The flow of cohesionless grains in fluids. Phil. Trans. R. Soc. Lond. 249, $235-297$. 
[5] Balay, S., Buschelman, K., Eijkhout, V., Gropp, W. D., Kaushik, D., Knepley, M. G., McInnes, L. C., Smith, B. F., Zhang, H., 2004. PETSc users manual. Tech. Rep. ANL-95/11 - Revision 2.1.5, Argonne National Laboratory.

[6] Balay, S., Buschelman, K., Gropp, W. D., Kaushik, D., Knepley, M. G., McInnes, L. C., Smith, B. F., Zhang, H., 2001. PETSc Web page. Http://www.mcs.anl.gov/petsc.

[7] Balay, S., Gropp, W. D., McInnes, L. C., Smith, B. F., 1997. Efficient management of parallelism in object oriented numerical software libraries. In: Arge, E., Bruaset, A. M., Langtangen, H. P. (Eds.), Modern Software Tools in Scientific Computing. Birkhäuser Press, pp. 163-202.

[8] Bercovier, M., Engelman, M., 1980. A finite-element method for incompressible non-newtonian flows. Journal of Computational Physics 36 (3), 313-326.

URL http: //www.sciencedirect.com/science/article/B6WHY-4DDR302-7K/1/a27540473088dcd2a4e158910bbbc4a4

[9] Bingham, E. C., 1922. Fluidity and Plasticity. McGraw Hill, New York.

[10] G. Carey and J. T. Oden. Finite Elements. Vol. VI: Fluids Mechanics. The Texas Finite Element Series, Prentice Hall, Englewood Cliffs, N.J., 1986.

[11] Charru, F., Mouilleron-Arnould, H., Eiff, O., 2004. Erosion and deposition of particles on a bed sheared by a viscous flow. Journal of Fluid Mechanics 519 (-1), 55-80.

[12] Dean, E. J., Glowinski, R., Guidoboni, G., 2007. On the numerical simulation of bingham visco-plastic flow: Old and new results. Journal of Non-Newtonian Fluid Mechanics 142 (1-3), 36-62.

URL http: //www.sciencedirect.com/science/article/B6TGV-4KV846H-1/2/7a6e1bcd0ee1a120c885591aae6cfc6a

[13] Einstein, H. A., 1950. The bed load function for sedimentation in open channel channel flows. Tech. Rep. 1026, U.S. Department of Agriculture.

[14] Fortin, M., Glowinski, R., 1983. Augmented Lagrangians : Application to the Numerica Solution of Boundary Value Problems. NorthHolland, Amsterdam.

[15] Fredsoe, J., Deigaard, R., 1992. Mechanics of costal sediment transport. word Scientific.

[16] Frigaard, I. A., Nouar, C., 2005. On the usage of viscosity regularisation methods for visco-plastic fluid flow computation. Journal of NonNewtonian Fluid Mechanics 127 (1), 1-26.

URL http: //www.sciencedirect.com/science/article/B6TGV-4G4XBV2-1/2/b071488697d73549ac617789b903a4c7

[17] Glowinsky, R., Le Tallec, P., 1989. Augmented Lagrangians and Operator-Splitting Methods in Nonlinear Mechanics. SIAM, Philadelphie.

[18] Goharzadeh, A., Khalili, A., Jorgensen, B. B., 2005. Transition layer thickness at a fluid-porous interface. Physics of Fluids 17 (5), 057102. URL http: //link. aip.org/link/?PHF/17/057102/1

[19] Guyon, E., Hulin, J.-P., Petit, L., 2001. Hydrodynamique physique. EDP Sciences - CNRS Editions, Paris.

[20] Happel, J., Brenner, H., 1973. Low Reynolds number hydrodynamics. Martinus Nijhof, The Hague.

[21] Jackson, R., 2000. The dynamics of fluidized particles. Cambridge University Press, Cambridge.

[22] Jop, P., Forterre, Y., Pouliquen, O., 2006. A constitutive law for dense granular flows. Nature 441, 727- 730.

URL http://dx.doi.org/10.1038/nature04801

[23] Lalli, F., Esposito, P. G., Piscopia, R., Verzicco, R., 2005. Fluid-particle flow simulation by averaged continuous model. Computers \& Fluids 34 (9), 1040-1061.

URL http: //www.science-direct.com/science/article/B6V26-4F3FDV3-2/1/59e809336e8c43572b2595b43f3a0631

[24] Mitsoulis, E., Zisis, T., 2001. Flow of bingham plastics in a lid-driven square cavity. Journal of Non-Newtonian Fluid Mechanics 101, $173-180$.

[25] J.T. Oden and G. Carey. Finite Elements. Vol. IV: Mathematical Aspects. The Texas Finite Element Series, Prentice Hall, Englewood Cliffs, N.J., 1986.

[26] Ouriemi, M., Aussillous, P., Guazzelli, E., 2009. Sediment dynamics. part i: Bed-load transport by shearing flows. Journal of Fluid Mechanics, in press. 
[27] Ouriemi, M., Aussillous, P., Guazzelli, E., 2009. Sediment dynamics. part ii: Dune formation in pipe flow. Journal of Fluid Mechanics, in press.

[28] Ouriemi, M., Aussillous, P., Medale, M., Peysson, Y., Guazzelli, E., 2007. Determination of the critical shields number for particle erosion in laminar flow. Physics of Fluids 19 (6), 061706.

URL http://link. aip.org/link/?PHF/19/061706/1

[29] Papanastasiou, T. C., 1987. Flows of materials with yield. Journal of Rheology 31, 385-404.

[30] O. Pironneau. Méthodes des éléments finis pour les fluides. Masson, Paris, 1988.

[31] Shields, A., 1936. Application of similarity principles and turbulence research to bed-load movement. Mitteilunger der Preussischen Versuchsanstalt fr Wasserbau und Schiffbau 26, 5-24.

[32] Yalin, S., 1963. An expression for bed-load transportation. J. Hydraul. Division HY3, 221-250.

[33] O. C. Zienkiewicz and R.L. Taylor The Finite Element Method. Fourth Edition, Volume 1, Mac Graw Hill, 1994.

\section{Figures}

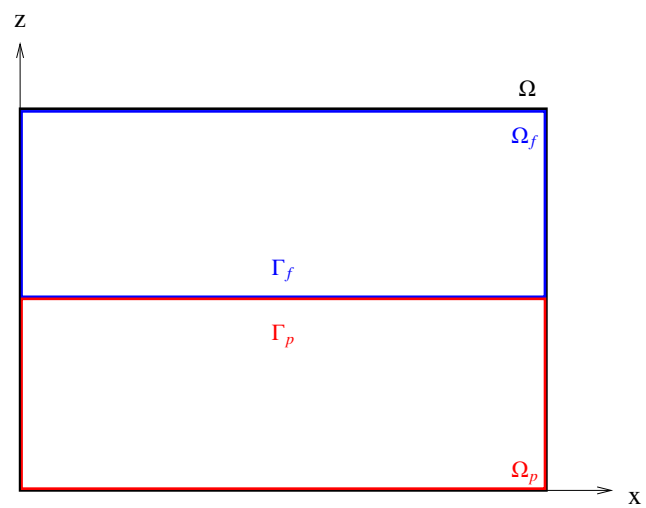

Figure 1: Sketch of domain definition for the fluid-particle problem.

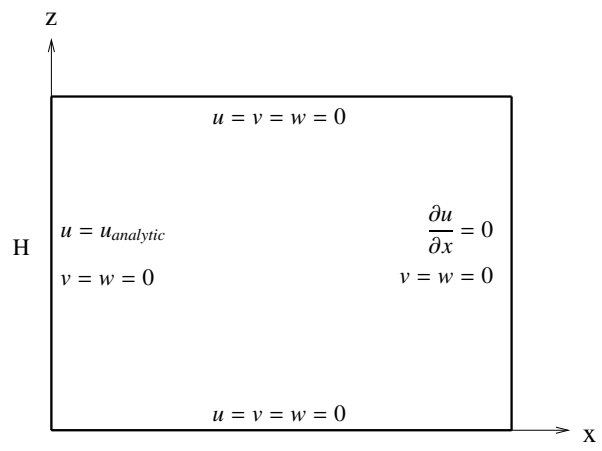

Figure 2: Sketch of the problem and boundary conditions of a Bingham fluid between two infinite parallel plane in 2D. 


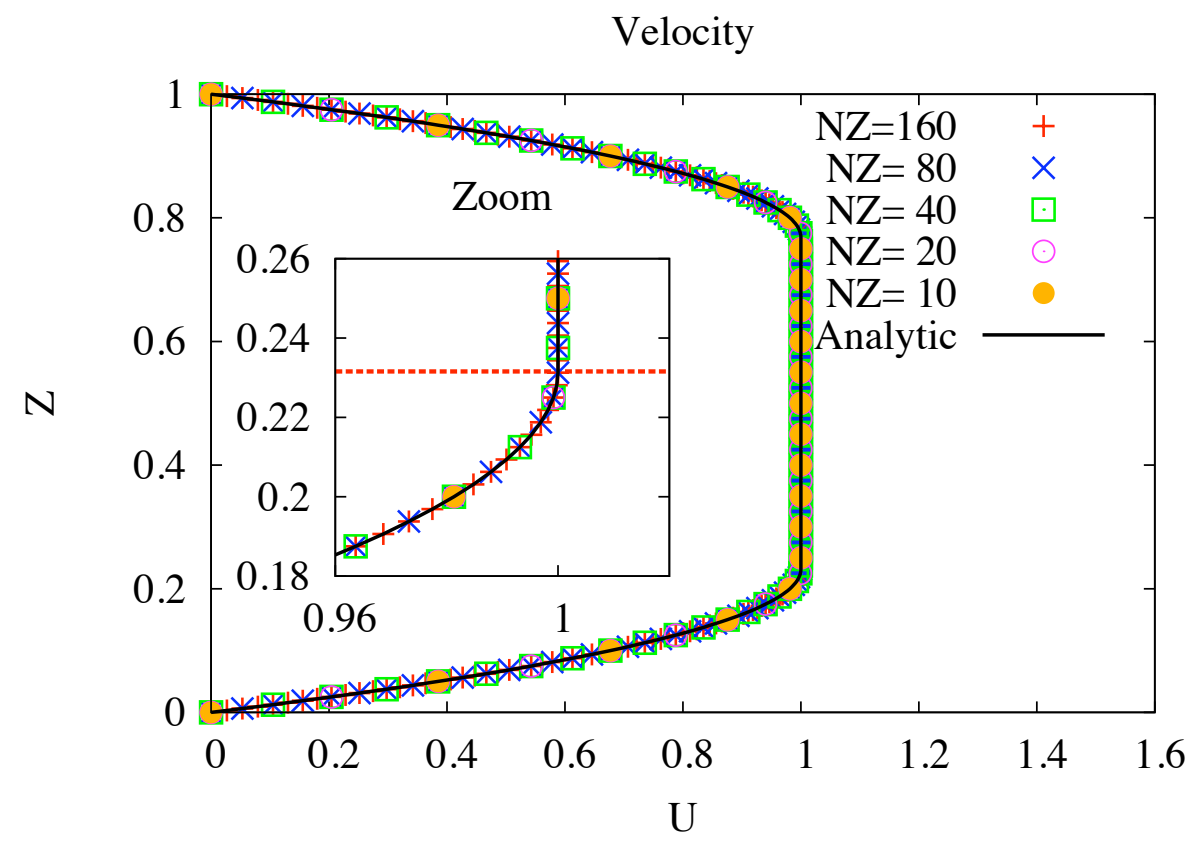

(a) Comparison of the longitudinal velocity profiles obtained by numerical simulations (symbols) for various meshes with the analytical solution (lines).

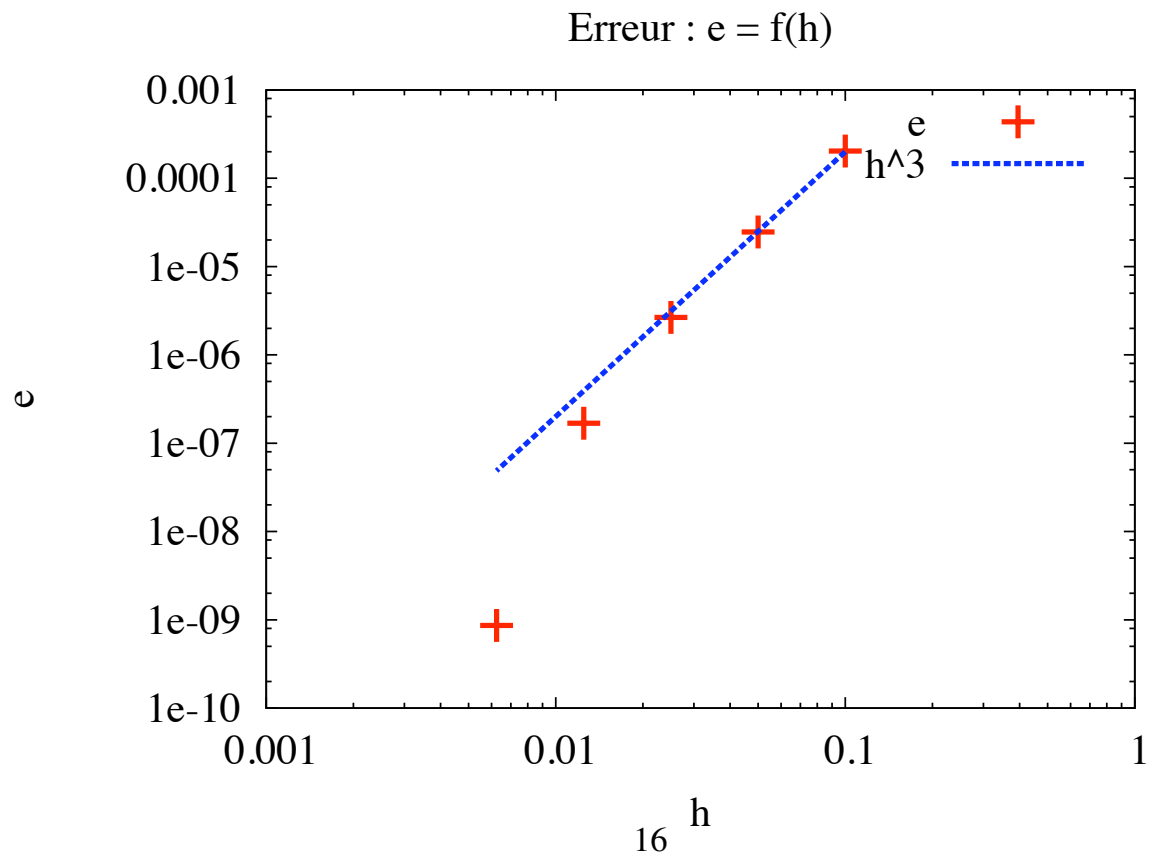

(b) Error analysis: RMS error against analytical solution $e=\frac{1}{N} \sqrt{\sum_{i=1}^{N}\left(U_{i}-U_{i}^{\text {ana }}\right)^{2}}$ where $N$ is the number of nodes. 


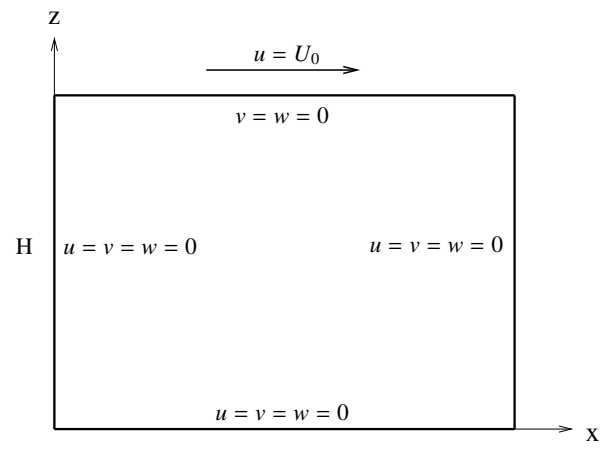

Figure 4: Sketch of the lid-driven cavity.

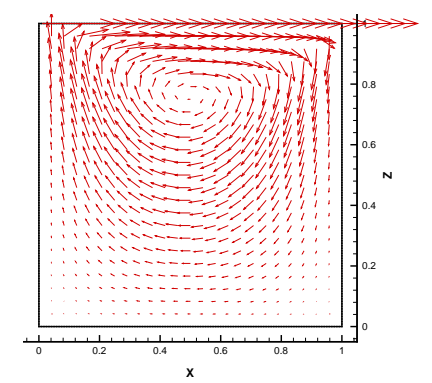

(a) $\mathrm{Bn}=0$

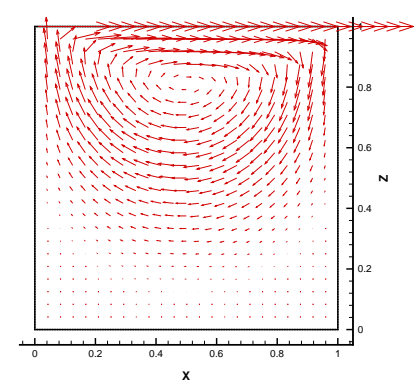

(b) $\mathrm{Bn}=2$

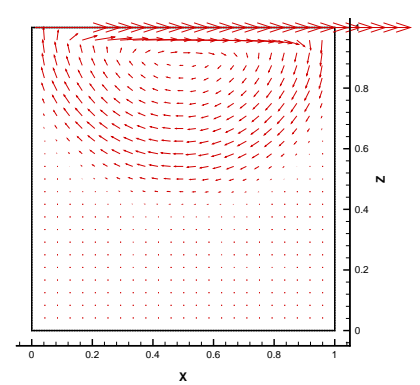

(c) $\mathrm{Bn}=20$

Figure 5: Velocity vector fields - Bingham varying from 0 to 20 


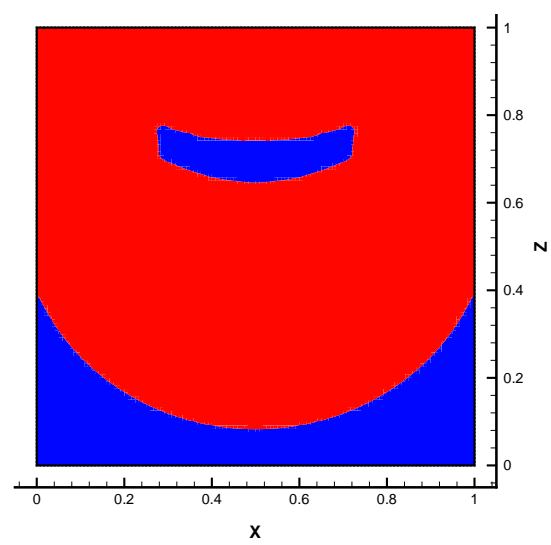

(a) Present model $-\mathrm{Bn}=2$

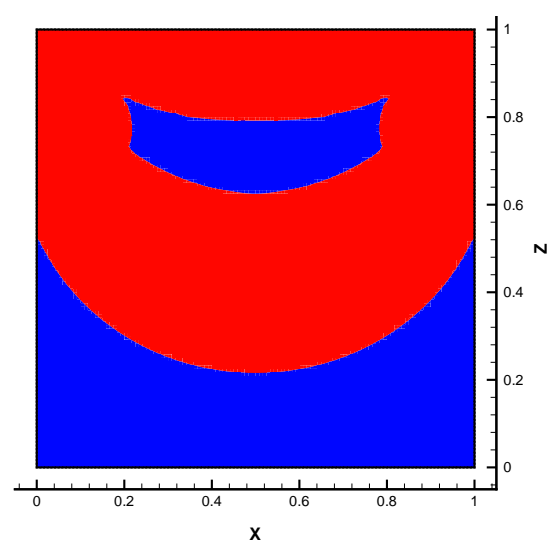

(c) Present model $-\mathrm{Bn}=5$

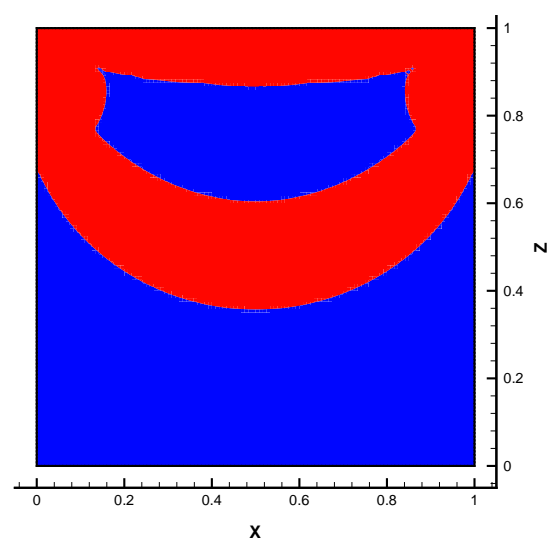

(e) Present model $-\mathrm{Bn}=20$

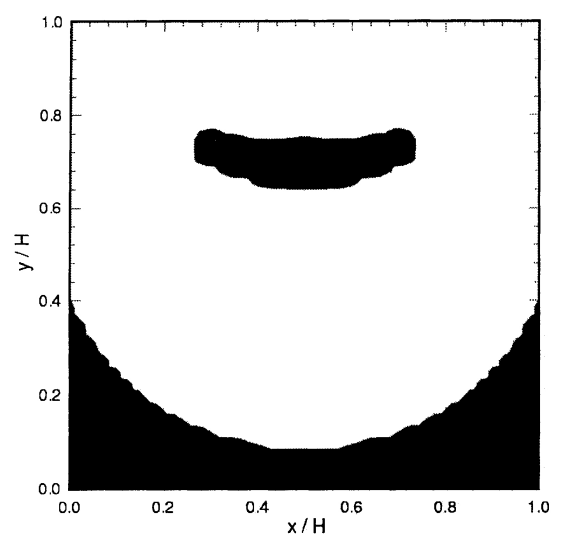

(b) Mitsoulis and Zisis (2001) [24] - Bn $=2$

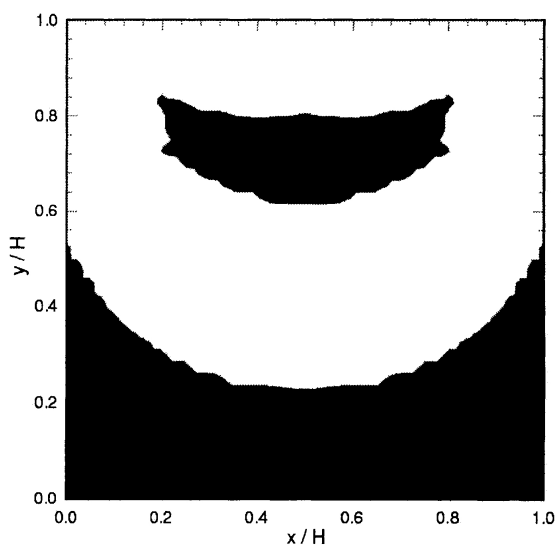

(d) Mitsoulis and Zisis (2001) [24] - Bn $=5$

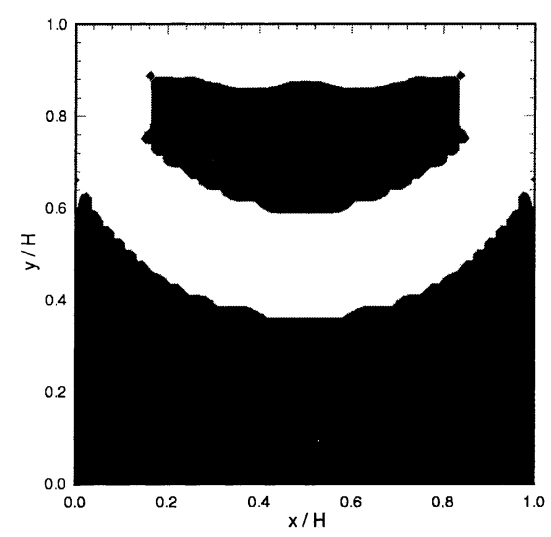

(f) Mitsoulis and Zisis (2001) [24] - Bn $=20$

Figure 6: Comparison of the present numerical model results with those from Mitsoulis and Zisis (2001) [24] for the yield zone position in the lid-driven cavity for various Bingham number $(\mathrm{Bn}=2,5$ and 20$)$. 


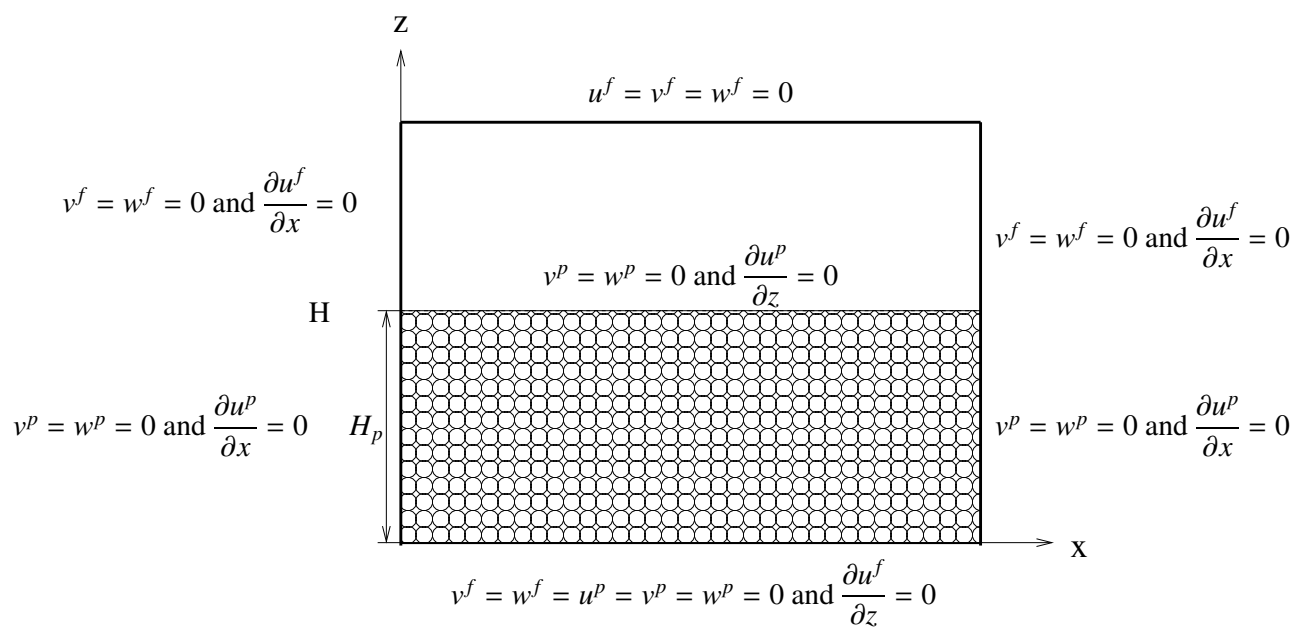

Figure 7: Sketch of the flow of a Newtonian fluid over a granular bed.

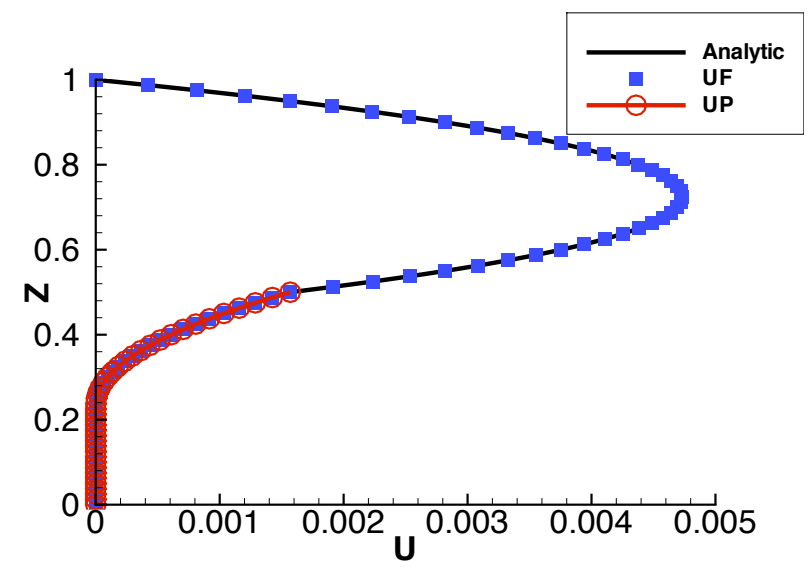

(a) Two-fluid model

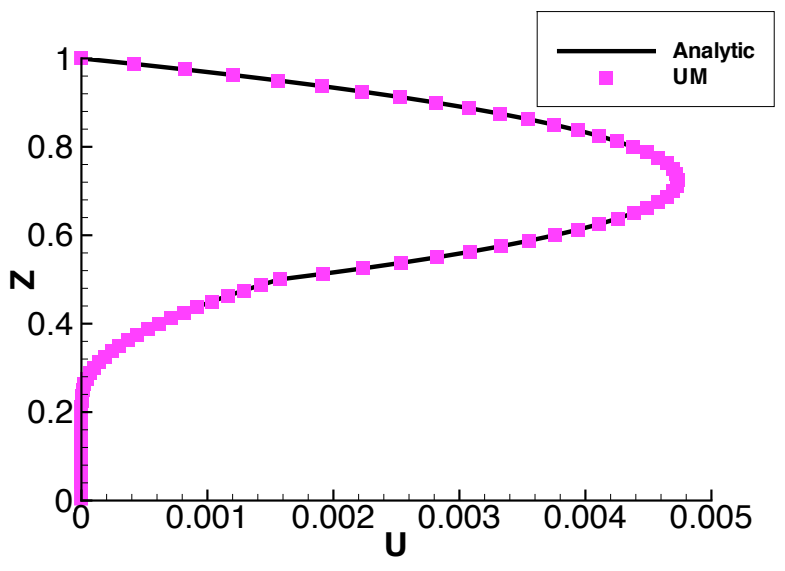

(b) Mixed-fluid model

Figure 8: Comparison of the longitudinal velocity profiles for the flow of a Newtonian fluid over a granular bed between two infinite parallel planes obtained by numerical simulations (two-fluid model) with the analytical solution of Ouriemi et al.[26]. 


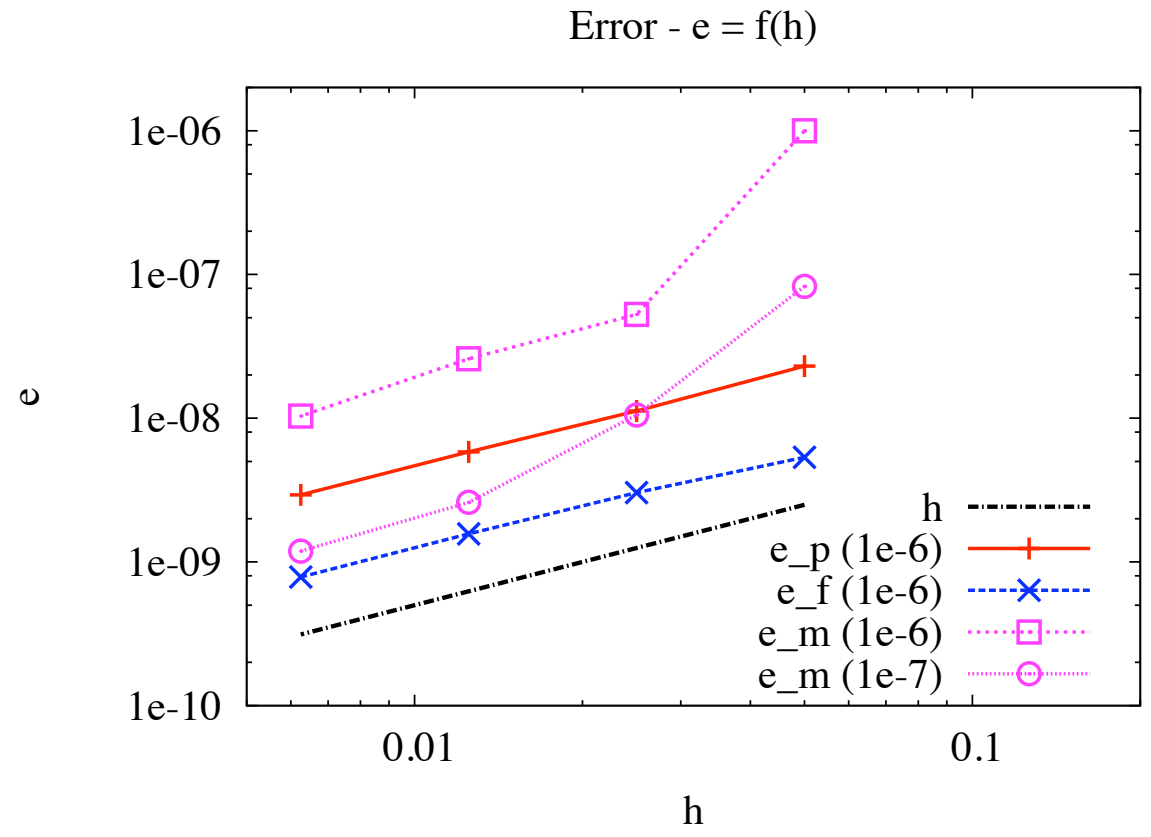

Figure 9: RMS Error against analytical solution for the flow of a Newtonian fluid over a moving granular bed between two infinite parallel planes: $e_{p}$ and $e_{f}$ stands for the particulate phase and the fluid phase error respectively for the two-fluid model whereas $e_{m}$ designates the mixture velocity error for the mixed-fluid model. The value in brackets is the value of the regularisation parameter $\lambda$. The RMS error is defined as: $e=\frac{1}{N} \sqrt{\sum_{i=1}^{N}\left(U_{i}-U_{i}^{\text {ana }}\right)^{2}}$ where $N$ is the number of nodes in the mesh. 


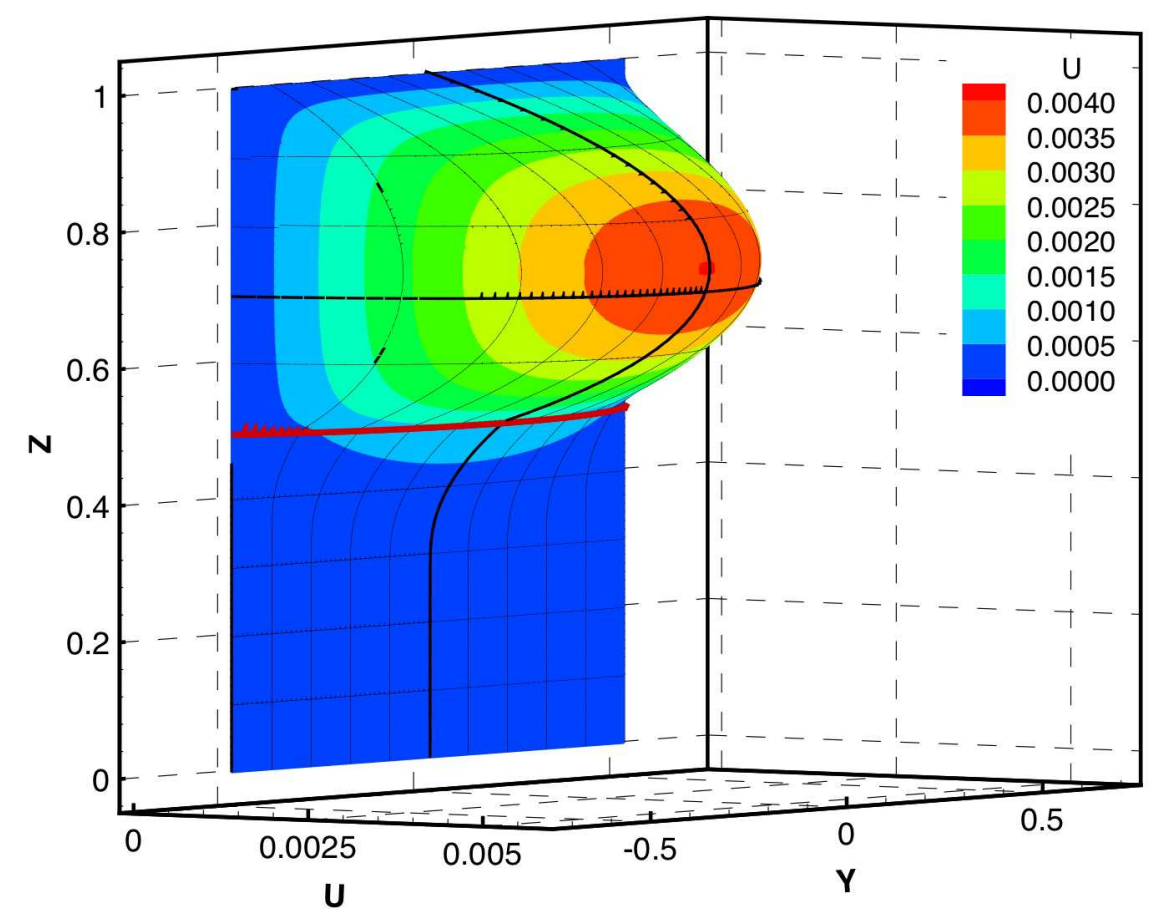

Figure 10: Velocity profile obtained by numerical simulations with the mixed-fluid model for the square cross-section duct (6x20x40).
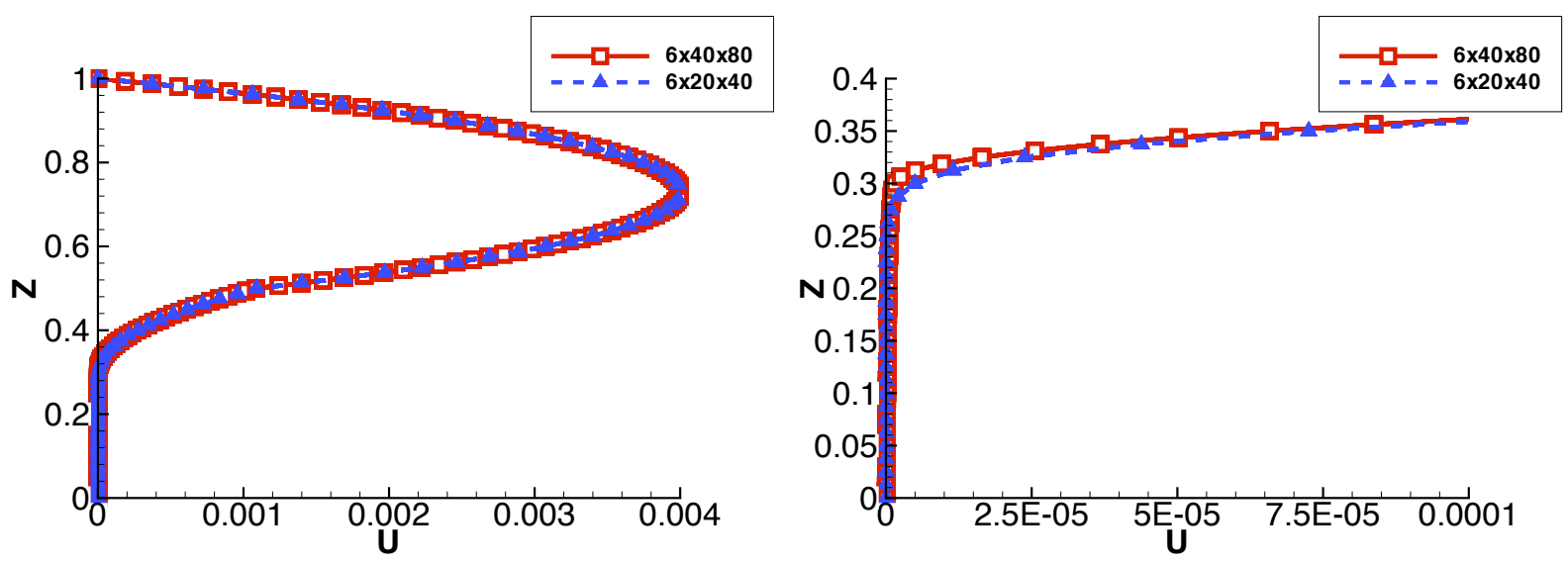

(a) Velocity profile in the plane of symmetry of the duct $(y=0)$

(b) Zoom on the yielded zone of the velocity profile in the plane of symmetry of the duct $(y=0)$

Figure 11: Mesh sensitivity of the velocity profiles obtained by numerical simulations with the mixed-fluid model for the square cross-section duct $(6 \times 20 \times 40$ and $6 \times 40 \times 80)$. 


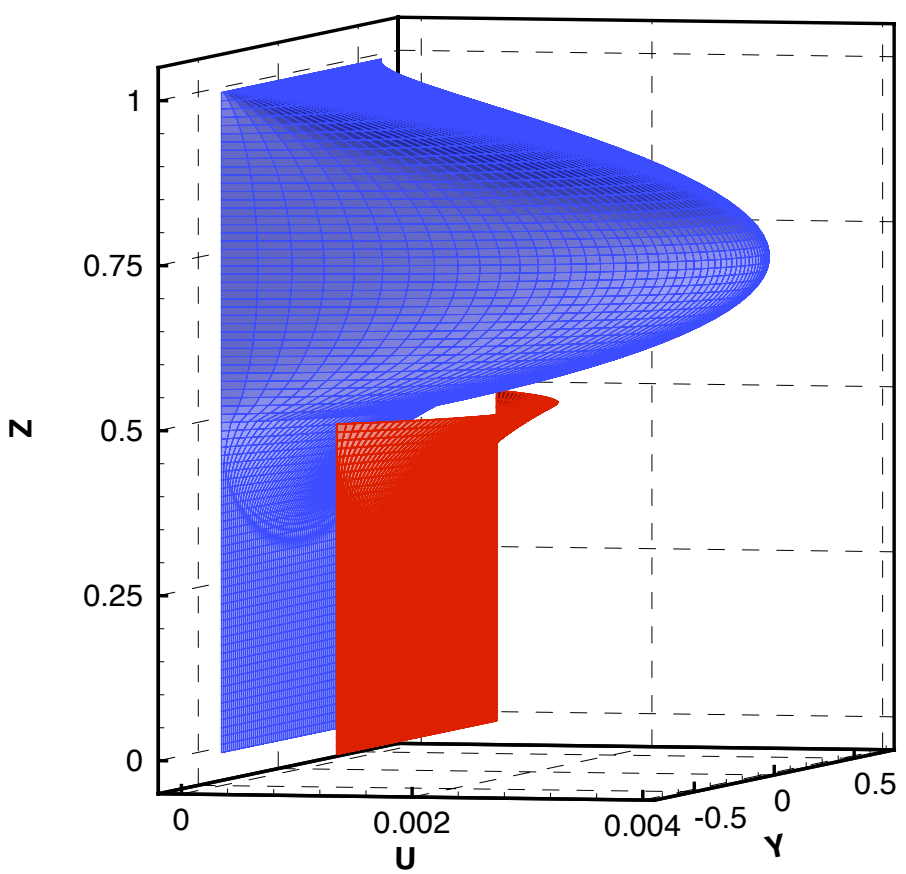

Figure 12: Velocity profile obtained by numerical simulations with the two-fluid model for the square cross-section duct (6x20x40). The fluid phase velocity is in blue and the particulate phase velocity is in red. An offset of $10^{-3}$ has been added to the velocity of the particulate phase (up) to make it visible.

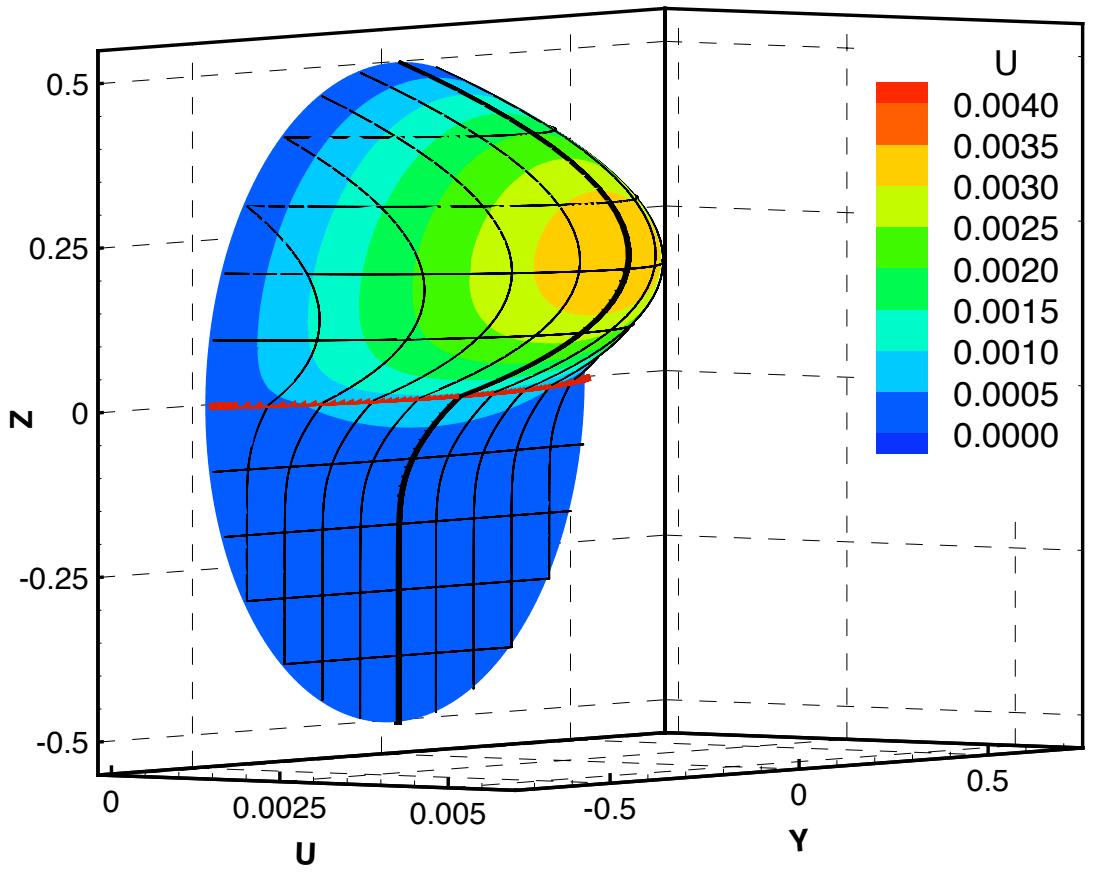

Figure 13: Velocity profile obtained by numerical simulations with the mixed-fluid model for the circular cross-section duct (6x896). 

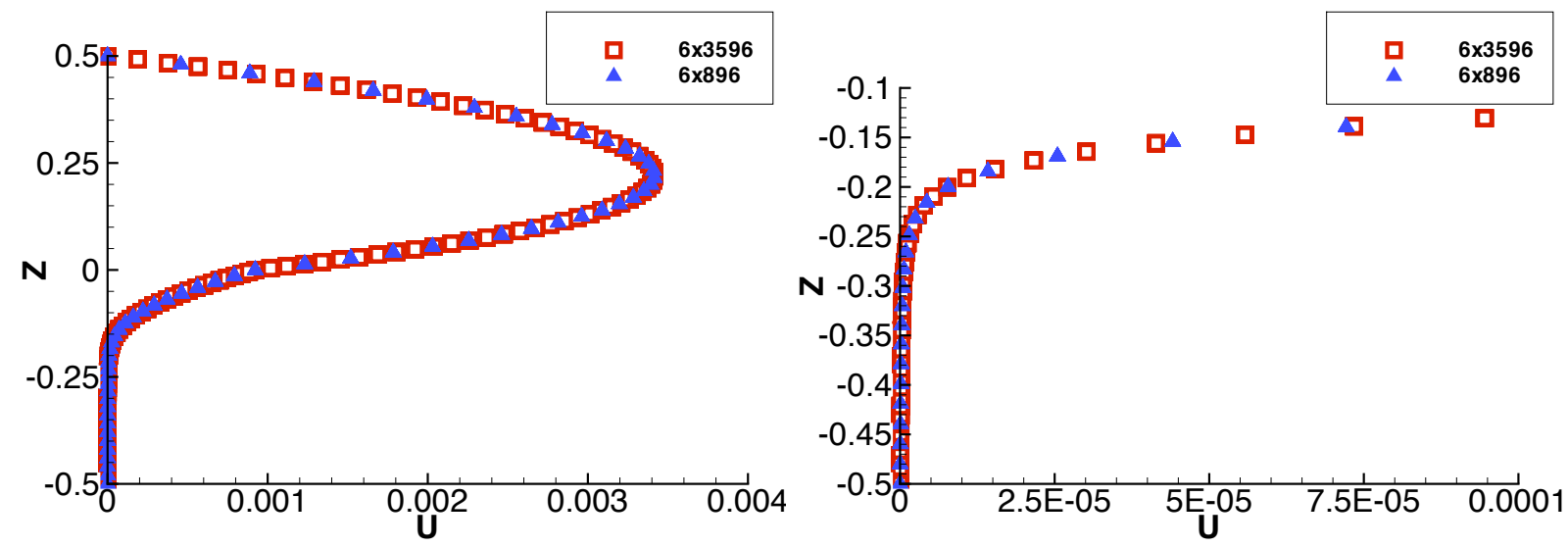

(a) Velocity profile in the plane of symmetry of the duct $(y=0)$

(b) Zoom on the yielded zone of the velocity profile in the plane of symmetry of the duct $(y=0)$

Figure 14: Mesh sensitivity of the velocity profiles obtained by numerical simulations with the mixed-fluid model for the square cross-section duct (6x896 and 6x3596).

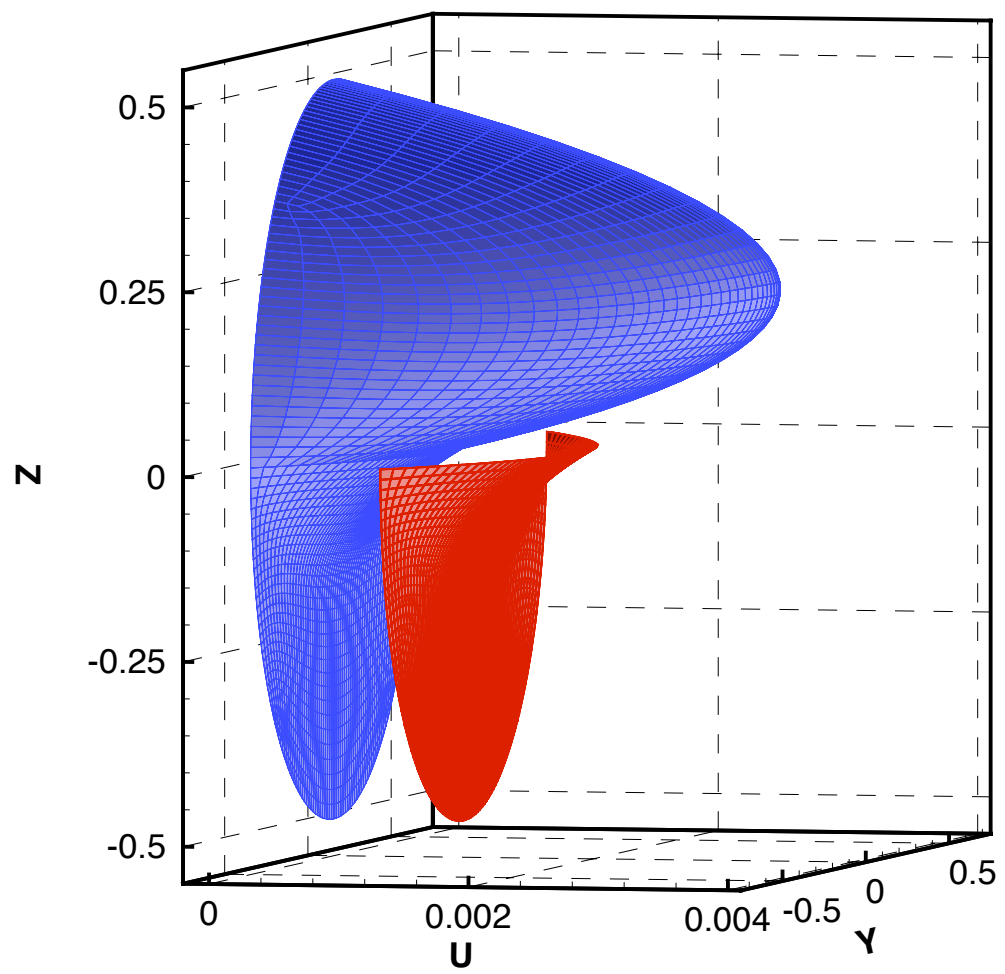

Figure 15: Velocity profile obtained by numerical simulations with the two-fluid model for the circular cross-section duct (6x896). The fluid phase velocity is in blue and the particulate phase velocity is in red. An offset of $10^{-3}$ has been added to the velocity of the particulate phase (up) to make it visible. 


\section{Tables}

\begin{tabular}{|c|c|c|c|c|c|}
\hline Mesh definition (NX x NY x NZ) & $3 \times 1 \times 10$ & $6 \times 1 \times 20$ & $12 \times 1 \times 40$ & $24 \times 1 \times 80$ & $48 \times 1 \times 160$ \\
\hline Degrees of freedom & 1323 & 4797 & 18225 & 71001 & 280233 \\
\hline CPU time (s/it. on 8 proc.) & 0.67 & 1.88 & 6.98 & 27.79 & 116.74 \\
\hline
\end{tabular}

Table 1: Mesh definition and CPU time per iteration

\begin{tabular}{|cc||c|c|}
\hline Mesh definition & NX x NY x NZ & $12 \times 1 \times 80$ & $24 \times 1 \times 160$ \\
\hline \hline Two-fluid model & DOF $^{1}$ & 54450 & 212562 \\
\cline { 2 - 4 }$\lambda=10^{-6}$ & Niter $^{2}$ & 1943 & 206 \\
\cline { 2 - 4 } & CPU time & $5682 \mathrm{~s}$ & $5476 \mathrm{~s}$ \\
\hline \hline Mixed-fluid model & DOF & 36225 & 141561 \\
\cline { 2 - 4 }$\lambda=10^{-6}$ & Niter & 269 & 54 \\
\cline { 2 - 4 } & CPU time & $438 \mathrm{~s}$ & $473 \mathrm{~s}$ \\
\hline \hline \multirow{2}{*}{$\lambda=10^{-7}$} & Niter & 253 & 75 \\
\cline { 2 - 4 } & CPU time & $453 \mathrm{~s}$ & $589 \mathrm{~s}$ \\
\hline
\end{tabular}

Table 2: CPU time and number of iterations 